\title{
Phase II study evaluating lapatinib in combination with $n a b$-paclitaxel in HER2-overexpressing metastatic breast cancer patients who have received no more than one prior chemotherapeutic regimen
}

\author{
Denise A. Yardley • Lowell Hart • Linda Bosserman • Mansoor N. Salleh • \\ David M. Waterhouse • Maura K. Hagan • Paul Richards • Michelle L. DeSilvio • \\ Janine M. Mahoney • Yasir Nagarwala
}

Received: 24 October 2012/ Accepted: 8 November 2012/Published online: 8 December 2012

(C) The Author(s) 2012. This article is published with open access at Springerlink.com

\begin{abstract}
Lapatinib, an oral, reversible inhibitor of epidermal growth factor receptor and human epidermal growth factor receptor 2 (HER2) tyrosine kinase, has proven antitumor activity in HER2-positive metastatic breast cancer (MBC). Nanoparticle albumin-bound paclitaxel (nab-paclitaxel) is indicated for the treatment of breast cancer after failure of combination chemotherapy for
\end{abstract}

This study was presented, in part, at the San Antonio Breast Cancer Symposium, San Antonio, TX, December 6-10, 2011; the 45th Annual Meeting of the American Society of Clinical Oncology, Orlando FL, May 29-June 2, 2009.

D. A. Yardley $(\bowtie)$

Sarah Cannon Research Institute, Nashville, TN, USA

e-mail: dyardley@tnonc.com

D. A. Yardley

Tennessee Oncology, PLLC, Nashville, TN, USA

L. Hart

Florida Cancer Specialists, Fort Myers, FL, USA

L. Bosserman

Willshire Oncology Medical Group, La Verne, CA, USA

M. N. Salleh

Georgia Cancer Specialists, Atlanta, GA, USA

D. M. Waterhouse

OHC (Oncology Hematology Care), Cincinnati, OH, USA

M. K. Hagan

Virginia Cancer Care, Mechanicsville, VA, USA

P. Richards

Oncology \& Hematology Associates of SW, Salem, VA, USA

M. L. DeSilvio - J. M. Mahoney - Y. Nagarwala

GlaxoSmithKline, Collegeville, PA, USA metastatic disease or relapse within 6 months of adjuvant chemotherapy. This was an open-label, single-arm, multicenter, Phase II study to evaluate the efficacy and safety of $n a b$-paclitaxel plus lapatinib in women with HER2 overexpressing MBC who had received no more than one prior chemotherapeutic regimen. The primary efficacy endpoint was the overall response rate (ORR). This was defined as the percentage of patients having either a complete response (CR) or partial response (PR). Secondary efficacy endpoints included progression-free survival (PFS), overall survival, duration of response (DoR), time to response (TTR), and time to progression (TTP). Investigator-assessed ORR was $53 \%(n=32,95 \%$ confidence interval (CI): 40.7-66.0) with the majority of patient responses demonstrating a PR (47\%). Four (7\%) patient responses demonstrated a CR, and ten $(17 \%)$ a stable disease. The median Kaplan-Meier estimate of investigator-assessed PFS, DoR, TTR, and TTP was 39.7 weeks (95\% CI 34.1-63.9), 48.7 weeks (95\% CI 31.7-57.1), 7.8 weeks (95\% CI 7.4-8.1), and 41 weeks (95\% CI 39.1-64.6), respectively. Lapatinib $1,000 \mathrm{mg}$ with nab-paclitaxel $100 \mathrm{mg} / \mathrm{m}^{2} \mathrm{IV}$ is feasible with manageable and predictable toxicity and an ORR of $53 \%$ comparing favorably with other HER2-based combinations in this setting.

Keywords Breast cancer · HER2 - Lapatinib . Nab-paclitaxel

\section{Introduction}

Breast cancer is the most commonly diagnosed tumor type in women in the United States with a significant proportion developing metastatic disease [1]. Metastatic breast cancer (MBC) is an incurable disease, and systemic treatment 
aims to prolong survival, control disease progression, alleviate symptoms, and enhance patient quality of life. Although early detection and improvements in therapy have augmented survival rates in recent years (5-year relative survival is $99 \%$ for localized disease and $84 \%$ for regional disease), the 5-year survival rates for patients with distant-stage disease remains low at $23 \%$ [1].

Control of several cellular processes in breast cancer is dependent on the epidermal growth factor receptor (EGFR, also known as ErbB) family, and members of this family have been proposed as targets in cancer therapy. Amplification of human epidermal growth factor receptor 2 (HER2/ErbB2) leads to increased receptor homo- and hetero-dimerization and subsequent activation of downstream signaling pathways associated with cell proliferation, differentiation, survival, and angiogenesis [2]. Initial studies indicated that $20-25 \%$ of tumors in women with early stage I-III breast cancers were HER2 positive [3, 4]. However, a larger and more recent study from Europe has reported HER2-positive breast cancer rates in the range of 13-19\% [5] which has both prognostic and predictive implications $[3,6]$.

HER2-positive tumors are associated with particularly aggressive disease and a poor prognosis with shorter overall survival (OS) and disease-free survival intervals [3]. Studies have shown that HER2-positive tumors are more likely to disseminate to major visceral sites as well as the central nervous system (CNS) [7, 8].

Trastuzumab, a monoclonal antibody directed against the HER2 receptor, has clinical activity in patients with HER2-positive breast carcinoma and is widely used in combination with cytotoxic agents [9-11]. A significant number of patients with HER2-positive MBC who are treated with trastuzumab experience symptomatic CNS metastases, potentially due to ineffective penetration of the CNS. Trastuzumab resistance may also develop, limiting trastuzumab's ability to maintain disease control in the advanced as well as early stage disease settings [12]. As such, alternative therapeutic strategies and combinations need to be developed to target HER2 signaling.

Lapatinib (Tykerb/Tyverb ${ }^{\circledR}$, GlaxoSmithKline) is an orally active small molecule that inhibits the tyrosine kinase activity of HER2 and EGFR. Lapatinib has shown clinical activity in combination with capecitabine in HER2positive tumors that progressed while on standard treatment, including trastuzumab [13]. Lapatinib, in combination with capecitabine was approved in 2007 by the U.S. Food and Drug Administration (US FDA) for the treatment of patients with advanced or MBC with HER2-positive tumors who have received prior therapy including an anthracycline, a taxane, and trastuzumab [14]. Lapatinib in combination with letrozole (an aromatase inhibitor) has also been shown to be effective in the treatment of postmenopausal women with hormone receptor-positive, HER2-positive MBC [15]. In 2010, the US FDA granted accelerated approval to lapatinib for use in combination with letrozole for the treatment of patients with HER2positive MBC and for whom hormonal therapy is indicated [14].

Nanoparticle albumin-bound paclitaxel (nab-paclitaxel; Abraxane ${ }^{\circledR}$ for Injectable Suspension, Celgene Corporation) is a novel Cremophor ${ }^{\circledR}$ EL-free, non-crystalline, amorphous, albumin-bound nanoparticle formulation of paclitaxel suspended in normal saline. It is associated with a lower risk of hypersensitivity as well as an improved toxicity profile, and has no requirements for premedication compared with solvent-based paclitaxel [16]. Furthermore, the neuropathy that frequently accompanies paclitaxel administration was easily managed and resolved more rapidly in patients treated with $n a b$-paclitaxel [16]. $\mathrm{Nab}$ paclitaxel demonstrated significantly longer progressionfree survival (PFS), compared with docetaxel, in patients with MBC $[17,18]$. Nab-paclitaxel has been approved by the US FDA in 2005 for the treatment of breast cancer after failure of combination chemotherapy for metastatic disease or relapse within 6 months of adjuvant chemotherapy. Prior therapy should have included an anthracycline unless clinically contraindicated [19].

In early as well as advanced HER2-positive breast cancer, synergy between chemotherapy and HER2-directed therapy (such as trastuzumab) has significantly improved outcomes, including survival, compared with chemotherapy alone $[10,11]$. However, despite treatment with taxane-based trastuzumab regimens in early stage disease, disease progressions are still evident. The proven activity of lapatinib in this setting (due to its differing mechanism of action) demonstrates that it is a suitable option in HER2positive MBC. Unlike trastuzumab, it has been suggested that lapatinib can cross the blood-brain barrier, providing a rationale for testing lapatinib in patients with CNS metastases [20,21]. Studies have also shown that HER3 can be transphosphorylated by p95 HER2 (a truncated version lacking the extracellular domain) in HER2-overexpressing breast cancer cell lines, and that this phosphorylation is inhibited by lapatinib, but not trastuzumab [22]. A more recent study demonstrated an OS benefit with lapatinib in combination with trastuzumab for patients with HER2positive MBC [23]. Also, owing to the toxicity of solventbased paclitaxel, evaluation of lapatinib in conjunction with $n a b$-paclitaxel for the treatment of HER2-positive MBC is warranted.

Presented here are the results of a single-arm, multicenter Phase II study undertaken to determine the activity of lapatinib plus $n a b$-paclitaxel in the first- and second-line setting in HER2-positive MBC. 


\section{Methods}

\section{Study design}

This was an open-label, single-arm, multicenter, Phase II study (NCT00709761) to evaluate the efficacy and safety of $n a b$-paclitaxel plus lapatinib in women with HER2-positive MBC who had received no more than one prior chemotherapeutic regimen in the metastatic setting. The planned enrollment was 60 patients with a lead in evaluation of the first 10 patients for safety of this combination. A safety analysis of the first ten enrolled patients treated for at least one cycle of the initial doses of $n a b$-paclitaxel $\left(125 \mathrm{mg} / \mathrm{m}^{2}\right.$ IV on Day 1,8 , and 15 every 28 days) in combination with lapatinib $(1,250 \mathrm{mg}$ orally once daily on a continuous basis) in a 4-week cycle for a planned minimum of six cycles was performed. However, during the ongoing safety review of the first five patients, Grade 3 toxicities were observed in all five patients (four with neutropenia and one with neutropenic fever and diarrhea) and the decision was made to reduce the dose of both study drugs. All subsequent patients $(n=55)$ received $n a b$-paclitaxel $\left(100 \mathrm{mg} / \mathrm{m}^{2}\right.$ IV on Day 1,8 , and 15 every 28 days) in combination with lapatinib $(1,000 \mathrm{mg}$ orally once daily on a continuous basis) in a 4-week cycle for a minimum of six cycles. If a complete response (CR) was obtained before six cycles, the patient had to receive two additional cycles of the combination regimen after which the CR was confirmed (a minimum of four cycles). After six cycles, patients continued to receive a daily dose of lapatinib $1,000 \mathrm{mg}$ orally until disease progression or withdrawal from study due to unacceptable toxicity or consent withdrawal. Patients could also continue to receive $n a b$-paclitaxel after six cycles if there was benefit without unacceptable toxicity.

Efficacy assessments were performed every 8 weeks and at the end of investigational treatment. All patients are being followed for survival. Lapatinib treatment delays of up to 2 weeks were permitted for resolution of toxicities, other than in the event of protocol defined liver abnormalities, left ventricular cardiac dysfunction, or interstitial pneumonitis. Only one dose reduction of both lapatinib (to $750 \mathrm{mg}$ ) and $n a b$ paclitaxel (to $80 \mathrm{mg} / \mathrm{m}^{2}$ ) was permitted for related toxicity.

Safety assessments were performed throughout the study and included physical examinations, Eastern Cooperative Oncology Group (ECOG) performance status (PS), vital signs, clinical laboratory evaluations, cardiac monitoring (left ventricular ejection fraction function at 12-week intervals for patients while receiving lapatinib), and recording of adverse events (AEs) and serious AEs (SAEs).

\section{Patient population}

Female patients $\geq 18$ years of age with histologically confirmed HER2-positive invasive breast cancer (defined as HER2 positive score [ $>2.2]$ by fluorescence in situ hybridization or $3+$ amplification by immunohistochemistry) who presented with de novo stage IV disease or had stage IV disease at a relapse after curative-intent surgery were enrolled in the study.

Patients were required to have received no more than one prior chemotherapeutic regimen in the metastatic setting. Prior endocrine therapy or trastuzumab treatment in the neoadjuvant, adjuvant, or metastatic setting was permitted. If a taxane had been previously administered, progression must have occurred $\geq 12$ months after completion of this treatment. Patients were also required to have an ECOG PS of 0 or 1, and measurable disease, according to Response Evaluation Criteria in Solid Tumors version 1.0 guidelines [24]. A cardiac ejection fraction of at least $50 \%$, as measured by echocardiogram or multigated acquisition scan, within the institutional range of normal was also essential. Patients with stable CNS metastases (stable for at least 3 months) were eligible if they were not taking steroids or enzyme-inducing anticonvulsants. Bisphosphonate therapy for bone metastases was allowed. However, treatment must have been initiated prior to the first dose of study medication. Key exclusion criteria included active cardiac, hepatic, or biliary diseases, concurrent treatment with other anticancer or investigational agents, peripheral neuropathy of Common Terminology Criteria for Adverse Events Grade 2 or greater, and diseases or surgeries affecting gastrointestinal function including malabsorption syndrome, gastric resection, and uncontrolled inflammatory bowel disease.

\section{Study endpoints}

The primary efficacy endpoint was overall response rate (ORR), based on confirmed responses from investigator assessment of best overall response. ORR was defined as the percentage of patients having either a CR or partial response (PR). Secondary efficacy endpoints included PFS (defined as time from the start of treatment until the earliest date of disease progression or death due to any cause, if sooner), OS (defined as time from the start of treatment until death due to any cause), duration of response (DoR) (defined for the subset of patients who show a CR or PRtime from first documented evidence of $\mathrm{CR}$ or PR until the first documented sign of disease progression or death due to any cause), and time to response (TTR) (defined for the subset of patients who show a CR or PR as time from the start of treatment until first documented evidence of PR or $\mathrm{CR}$, whichever is recorded first). When tumor response was confirmed at a repeat assessment, the TTR was taken to be the first time the response was observed. Time to progression (TTP) (defined as time from the start of treatment 
until the earliest date of disease progression, or death due to breast cancer, if sooner) was also included as another secondary efficacy endpoint.

\section{Statistical analyses}

All efficacy analyses were conducted on the intent-to-treat population, which comprised all patients who received at least one dose of study drug. Exact binomial $95 \%$ confidence interval (CI) for the overall response rate was used. The Kaplan-Meier estimate for the median PFS, OS, DoR, TTR, and TTP were summarized, along with approximate $95 \%$ CI, if there were a sufficient number of events. The estimate of the standard error was computed by Greenwood's formula [25]. The study was not designed for inference testing. Sample-size considerations were based entirely on feasibility. No statistical hypotheses were tested, and the focus was to estimate the ORR who received no more than 1 prior treatment for HER2-positive MBC. A sample size of 60 was targeted to insure that the lower limit of the $95 \% \mathrm{CI}$ exceeded $50 \%$ for the overall response rate.

\section{Results}

Patient population

From July 2, 2008 to January 5, 2011, 60 patients were enrolled and treated at 14 centers in the United States. Patient demographics and prior anticancer treatments are summarized in Table 1. The median time from initial diagnosis (any stage) was 28.9 months; $25 \%$ of the patients had previously received one prior regimen in the metastatic setting and $57 \%$ of patients had previously received neo/adjuvant therapy. Disease burden at baseline is shown in Table 2. The most common sites of metastatic disease were lymph nodes, followed by the lung, liver, bone, and breast.

Primary efficacy results: overall tumor response rate

Lapatinib $+n a b$-paclitaxel was efficacious with respect to investigator-assessed $\mathrm{ORR} \quad(53 \% \quad[n=32, \quad 95 \% \mathrm{CI}$ 40.7-66.0]) with the majority of patients having a PR (28; $47 \%$ ), four patients (7\%) a CR and ten patients (17\%) showing stable disease (SD) (Table 3). A total of ten patients had unknown responses. These were patients who withdrew on or before the first response assessment time point of 8 weeks with no tumor assessments available with the exception of one patient who withdrew at week 9 due to diarrhea and refused to have the bone scans.
Secondary efficacy results

The median Kaplan-Meier estimate of investigator-assessed PFS was 39.7 weeks (95\% CI 34.1-63.9) (Table 3). As of the data cut-off, 13 patients (22\%) had died. Therefore, the median OS was not reached as the data were not mature (i.e., $>75 \%$ of the patients were censored for the endpoint). The median Kaplan-Meier estimate of investigator-assessed TTR and DoR was 7.8 weeks (95\% CI 7.4-8.1) and 48.7 weeks (95\% CI 31.7-57.1) for patients who responded (CR or PR). The Kaplan-Meier estimate for median TTP was 41 weeks (95\% CI 39.1-64.6). TTP was similar to PFS in this study as the majority of PFS events (either progression or death due to any cause) were disease progressions (28 out of 30 ). Two PFS events were deaths due to causes other than breast cancer and were considered a competing risk for the TTP analysis.

\section{Lapatinib and nab-paclitaxel exposure}

The median duration of exposure to lapatinib was 5.6 months (range, 0-19 months). The mean daily lapatinib dose was $893.1 \mathrm{mg}$. Approximately half of the study population (31 patients; $52 \%$ ) completed six cycles of treatment with nab-paclitaxel, which was the duration of treatment specified in the protocol. A total of 13 patients (22\%) continued to participate in the study for further cycles of treatment. In total, 73 lapatinib dose interruptions occurred in 34 patients ( $57 \%$ ) were of short duration (51/ 73 interruptions were $\leq 7$ days in duration), and were mainly due to non-hematologic toxicities (52\%). Twentysix reductions in the dose of lapatinib were made in 24 patients $(40 \%)$ and were also mainly due to non-hematologic toxicities $(85 \%)$. Twenty-four doses of nab-paclitaxel were delayed in 15 patients $(25 \%)$, and were most commonly due to non-hematologic toxicity (42\%). Sixteen dose reductions of nab-paclitaxel occurred in 13 patients $(22 \%)$ mainly due to hematologic toxicities (56\%). Doses of nab-paclitaxel were missed by 33 patients (55\%) and the majority of these patients missed one or two doses (53 missed doses in total). The most common reason for missing a dose was non-hematologic toxicity $(47 \%)$.

\section{Safety}

The most commonly reported AEs were diarrhea, fatigue, nausea, and rash (Table 4). The maximum toxicity grade of the majority of frequently reported AEs was Grade 1 or 2. Grade 3 diarrhea was reported at a frequency of $20 \%$, contributing to a high overall frequency $(62 \%)$ of AEs with a maximum toxicity Grade of 3 . AEs of neutropenia also reached a maximum toxicity Grade of 3 for $22 \%$ of 
Table 1 Patient demographics and prior anticancer therapies

\begin{tabular}{lc}
\hline Characteristic & $\begin{array}{c}\text { Lapatinib }+n a b- \\
\text { paclitaxel }(n=60)^{\mathrm{a}}\end{array}$ \\
\hline Age, years & \\
Median (range) & $56(28-80)$ \\
HER2 status, $n(\%)$ & $37(62)$ \\
FISH+ (with or without IHC+) & $23(38)$ \\
IHC 3+ (only) & \\
ER/PgR status, $n(\%)$ & $34(57)$ \\
ER+ and/or PgR+ & $26(43)$ \\
ER- and PgR- & \\
Line of metastatic treatment, $n(\%)$ & $45(75)$ \\
First-line & $15(25)$ \\
Second-line & 28.9 \\
Median time since first diagnosis, months & $34(57)$ \\
Prior anticancer therapy, $n(\%)$ & \\
Chemotherapy & $31(52)$ \\
Neo-/adjuvant & $8(13)$ \\
Metastatic & $4(7)$ \\
Neo-/adjuvant/metastatic & $10(17)$ \\
Taxanes & $22(37)$ \\
Neo-/adjuvant & $4(7)$ \\
Metastatic & $2(3)$ \\
Neo-/adjuvant/metastatic & \\
Hormonal & \\
Neo-/adjuvant & \\
Metastatic & \\
Neo-/adjuvant/metastatic & \\
Trastuzumab & \\
Neo-/adjuvant & \\
Metastatic & \\
Neo-adjuvant/metastatic & \\
\hline
\end{tabular}

ER estrogen receptor, FISH fluorescence in situ hybridization, HER2 Human epidermal growth factor receptor 2, IHC immunochemistry, $P g R$ progesterone receptor

a All patients were female

Table 2 Disease burden at time of study enrollment

\begin{tabular}{ll}
\hline & $\begin{array}{l}\text { Lapatinib }+n a b- \\
\text { paclitaxel }(n=60)\end{array}$ \\
\hline Number of organs involved, $n(\%)$ & $12(20)$ \\
1 & $25(42)$ \\
2 & $23(38)$ \\
$\geq 3$ & \\
Sites of disease, $n(\%)$ & $38(63)$ \\
Lymph nodes & $32(53)$ \\
Lung & $24(40)$ \\
Liver & $16(27)$ \\
Bone & $12(20)$ \\
Breast (de novo Stage IV) & \\
\hline
\end{tabular}

patients. A total of five patients ( $8 \%$ ) experienced Grade 4 events (diarrhea, nausea, fatigue, febrile neutropenia, and hypokalemia) and all these AEs were considered treatmentrelated.

Eighteen patients $(30 \%)$ reported SAEs (Table 5). The most frequently reported SAEs were dehydration $(n=3$; $5 \%)$ and diarrhea $(n=3 ; 5 \%)$.

Forty-nine patients $(82 \%)$ discontinued study treatment, predominantly due to disease progression (28 patients), and $10 / 49$ patients $(17 \%$ ) experienced an AE that led to the permanent discontinuation of study treatment (Table 6). Overall, 13 deaths were reported in this study with the primary cause of death due to breast cancer ( $n=10 / 13$; $17 \%$ ). Two patients (3\%) died due to SAEs (sudden death, acute renal failure) which were considered to be related to treatment. There were no clinically significant changes in vital signs, body weight, and ECOG PS during the study.

\section{Discussion}

The primary objective of this study was to determine the ORR in patients with HER2-positive MBC who received first-line or second-line treatment with lapatinib in combination with weekly nab-paclitaxel. This study was not designed for inference testing thus definitive conclusions cannot be derived from the results. A clinical benefit was observed for treatment with lapatinib plus nab-paclitaxel with respect to investigator-assessed ORR in 32 patients (53\%). The highest number of responses was PR (28 patients; $47 \%$ ). Four patients $(7 \%)$ had a CR and ten patients $(17 \%)$ showed SD.

ORR in this study was lower compared with other studies conducted to investigate paclitaxel and lapatinib combination therapy. In a Phase III, multicenter, doubleblind, placebo-controlled study in which patients with HER2-positive MBC (Stage IV) received treatment with paclitaxel and either lapatinib or placebo (NCT00281658), ORR was $69 \%$ [26].

In a Phase II study in which 57 female patients with HER2-positive MBC (Stage IV) were randomized to receive paclitaxel and lapatinib (NCT00356811) ORR was $77 \%$ [27]. These differences could be due in part to the small sample size, confounded by the inclusion of both first-line and second-line patients and the fact that ten patients in this study had an unknown response (no tumor assessments available).

The clinically meaningful activity observed in the primary endpoint was supported by investigator-assessed PFS, where the median Kaplan-Meier estimate of PFS was 39.7 weeks. These results are consistent with those of other trials evaluating paclitaxel and lapatinib combination 
Table 3 Efficacy results (ITT population)

\begin{tabular}{ll}
\hline Characteristic & $\begin{array}{l}\text { Lapatinib }+n a b- \\
\text { paclitaxel }(n=60)\end{array}$ \\
\hline
\end{tabular}

Overall response rate (ORR)

Response rate (CR + PR), $n(\%)(95 \%$ CI) 32 (53) (40.7-66.0)

Best response, $n(\%)$

$\begin{array}{ll}\mathrm{CR} & 4(7) \\ \mathrm{PR} & 28(47) \\ \mathrm{SD} & 10(17) \\ \mathrm{PD} & 8(13)\end{array}$

$\begin{array}{lc}\text { Unknown } & 10(17)^{\mathrm{a}} \\ \text { PFS } & \\ \text { Patients, } n(\%) & 60(100)\end{array}$

Progressed or died due to any cause $\quad 30(50)$

Censored, follow-up ended 19 (32)

Censored, follow-up ongoing $11(18)^{\mathrm{b}}$

Kaplan-Meier estimate for PFS (weeks)

Median (95\% CI)

TTR

Patients, $n(\%)$

Kaplan-Meier estimate for TTR (weeks)

Median (95\% CI)

DoR

Patients, $n(\%)$

Progressed or died due to any cause

Censored, follow-up ended

Censored, follow-up ongoing

Kaplan-Meier estimate for DoR (weeks)

Median (95\% CI)

TTP

Patients, $n(\%)$

Progressed or died due to breast cancer

Censored, died due to other cause

Censored, follow-up ended

Censored, follow-up ongoing

Kaplan-Meier estimate for TTP (weeks)

Median (95\% CI)

$39.7(34.1-63.9)$

32 (53)

$7.8(7.4-8.1)$

$32(53)$

17 (53)

4 (13)

$11(34)^{\mathrm{b}}$

$48.7(31.7-57.1)$

$60(100)$

$28(47)$

2 (3)

19 (32)

$11(18)^{\mathrm{b}}$

$41.0(39.1-64.6)$

$C I$ confidence interval, $C R$ complete response, DoR duration of response, ITT intent-to-treat, $P D$ progressive disease, $P F S$ progression-free survival, $P R$ partial response, $S D$ stable disease, TTR time to response, TTP time to progression

${ }^{a}$ Patients who withdrew before the first response assessment. Patients with unknown responses were treated as non-responders

b At the time of this reporting, there were 11 patients still ongoing on lapatinib monotherapy treatment

therapy. In two similar studies, the median PFS were 9.7 months (41.7 weeks) (NCT00281658) [26] and 47.9 weeks (NCT00356811) [27].

The median duration of lapatinib and $n a b$-paclitaxel treatment was 24.3 and 24 weeks, respectively. These data are comparable to results from a randomized, multicenter,
Table 4 AEs with $>15 \%$ incidence by maximum toxicity grade (ITT population)

\begin{tabular}{|c|c|c|c|c|}
\hline \multirow[t]{3}{*}{ Adverse events } & \multicolumn{4}{|c|}{ Number of subjects $(\%)$} \\
\hline & \multicolumn{4}{|c|}{ Lapatinib + nab-paclitaxel $(n=60)$} \\
\hline & Grade 1 & Grade 2 & Grade 3 & Grade 4 \\
\hline Diarrhea $(n=54)$ & 38 & 30 & 20 & 2 \\
\hline Fatigue $(n=41)$ & 40 & 18 & 8 & 2 \\
\hline Nausea $(n=39)$ & 38 & 23 & 2 & 2 \\
\hline $\operatorname{Rash}(n=29)$ & 25 & 18 & 5 & 0 \\
\hline Alopecia $(n=24)$ & 15 & 25 & 0 & 0 \\
\hline Vomiting $(n=23)$ & 25 & 8 & 5 & 0 \\
\hline Anemia $(n=22)$ & 17 & 18 & 2 & 0 \\
\hline Neutropenia $(n=22)$ & 5 & 10 & 22 & 0 \\
\hline $\begin{array}{l}\text { Neuropathy peripheral } \\
\quad(n=21)\end{array}$ & 20 & 12 & 3 & 0 \\
\hline Edema peripheral $(n=16)$ & 15 & 12 & 0 & 0 \\
\hline Cough $(n=15)$ & 20 & 5 & 0 & 0 \\
\hline Dyspnea $(n=15)$ & 15 & 5 & 5 & 0 \\
\hline Decreased appetite $(n=15)$ & 10 & 13 & 2 & 0 \\
\hline Constipation $(n=13)$ & 20 & 2 & 0 & 0 \\
\hline Epistaxis $(n=13)$ & 22 & 0 & 0 & 0 \\
\hline Nail disorder $(n=13)$ & 13 & 8 & 0 & 0 \\
\hline Dehydration $(n=12)$ & 0 & 13 & 7 & 0 \\
\hline Weight decreased $(n=11)$ & 10 & 7 & 2 & 0 \\
\hline Pyrexia $(n=11)$ & 15 & 3 & 0 & 0 \\
\hline Insomnia $(n=11)$ & 15 & 3 & 0 & 0 \\
\hline Dyspepsia $(n=10)$ & 15 & 2 & 0 & 0 \\
\hline Pain in extremity $(n=10)$ & 12 & 5 & 0 & 0 \\
\hline Dysgeusia $(n=10)$ & 13 & 3 & 0 & 0 \\
\hline
\end{tabular}

$A E$ adverse event, $I T T$ intent-to-treat

double-blind, placebo-controlled, 2-Arm, Phase III Study of lapatinib in combination with paclitaxel in subjects previously untreated for advanced or MBC (NCT0007527 0) [28]. In this study, the median duration of lapatinib treatment was 19.9 weeks, and the median duration of paclitaxel treatment was 15.1 and 16.1 weeks in the paclitaxel-lapatinib and paclitaxel-placebo arms, respectively.

The maximum toxicity grade of the majority of AEs was Grade 3 or less, and most resolved without sequelae. For hematologic toxicities, Grade 3 neutropenia and anemia was observed in 22 and $2 \%$ of patients, respectively, which was lower compared with 35 and $4 \%$ observed in the lapatinib plus paclitaxel study (NCT00281658) [26]. However, the non-hematologic toxicities observed in this study were consistent with the NCT00281658 study [26]. Grade 3 diarrhea, rash, and fatigue were reported by 20,5 , and $8 \%$ of the patients, respectively, in this study compared with 20, 4, and $2 \%$ in the NCT00281658 study. Although Grade 3 AEs of diarrhea and neutropenia were reported by 20 and $22 \%$ of patients, respectively, low numbers of patients withdrew due to these AEs ( $7 \%$ due to 
Table 5 Serious adverse events (SAEs)

\begin{tabular}{ll}
\hline & $\begin{array}{l}\text { Lapatinib }+n a b- \\
\text { paclitaxel }(n=60)\end{array}$ \\
\hline Any SAE, $n(\%)$ & $18(30)$ \\
With $\geq 2(3 \%)$ patients, $n(\%)$ & \\
Dehydration & $3(5)$ \\
Diarrhea & $3(5)$ \\
Anemia & $2(3)$ \\
Cellulitis & $2(3)$ \\
Febrile neutropenia & $2(3)$ \\
Hypokalemia & $2(3)$ \\
Renal failure acute & $2(3)$ \\
Drug-related fatal SAEs, $n(\%)$ & $2(3)^{\mathrm{a}}$ \\
\hline
\end{tabular}

Irrespective of relationship to study treatment

a One patient (lapatinib $750 \mathrm{mg} / \mathrm{day}$, nab-paclitaxel $100 \mathrm{mg} / \mathrm{m}^{2}$ weekly) with a history of arrhythmia experienced a sudden death of presumed cardiac origin. One patient (lapatinib 1,000 mg/day, nabpaclitaxel $100 \mathrm{mg} / \mathrm{m}^{2}$ weekly) with uncontrolled diabetes developed acute renal failure and dehydration

Table 6 Study treatment overview

\begin{tabular}{ll}
\hline & $\begin{array}{l}\text { Lapatinib }+n a b- \\
\text { paclitaxel }(n=60)\end{array}$ \\
\hline Discontinued study treatment, $n(\%)$ & $49(82)$ \\
Ongoing, $n(\%)$ & $11(18)^{\mathrm{a}}$ \\
Reason for discontinuation, $n(\%)$ & \\
Disease progression & $28(47)$ \\
Adverse events & $10(17)$ \\
Decision by patient & $6(10)$ \\
Protocol deviation $^{\mathrm{b}}$ & $3(5)$ \\
Investigator decision & $2(3)$
\end{tabular}

$\bar{a}$ At the time of this reporting, there were 11 patients continuing on the lapatinib monotherapy

b Two patients off study treatment for longer than the protocol defined 2 weeks; one patient received radiation to the right breast while on study

diarrhea, none due to neutropenia). SAEs were reported by $30 \%$ of patients. The most frequently reported SAEs were dehydration $(5 \%)$ and diarrhea (5\%). Two patients died during the study due to SAEs (sudden death in a patient with a medical history of cardiac arrhythmias, and acute renal failure in a patient with a medical history of uncontrolled diabetes).

This study established a dose regimen of $n a b$-paclitaxel $\left(100 \mathrm{mg} / \mathrm{m}^{2}\right.$ IV on Day 1,8 , and 15 every 28 days) in combination with lapatinib $(1,000 \mathrm{mg}$ orally once daily on a continuous basis) in a 4-week cycle as a feasible treatment for HER2-positive MBC with manageable and predictable toxicity. The incidence and severity of AEs for the combination treatment was considered to be consistent with the known safety profiles of lapatinib and nab-paclitaxel. Overall, the data in this study are consistent with those reported for other studies of lapatinib in combination with paclitaxel. Although this study was not designed for inference testing, the results suggest a promising signal of efficacy and no new safety signals.

Acknowledgments We would like to thank all of our patients and their family members for participating in this clinical trial and Celgene/Abraxis BioScience for providing the nab-paclitaxel for this clinical trial. LTP111111 investigators: Robert F. Asbury, Evergreen Hematology \& Oncology, Spokane, Washington; Min-Chun Chen, Multicare Health System, Tacoma, Washington; Ellen Chuang, Weill Medical College of Cornell University New York, New York; James Congdon, Providence Everett Medical Center, Everett, Washington; Kiem D. Liem, Pacific Shores Medical Group, Long Beach, California; Paula Silverman, University Hospitals of Cleveland, Cleveland, Ohio; Jacqueline Vuky, Virginia Mason Medical Center, Seattle, Washington. Editorial and medical writing support was provided by Sarah White and Karen Yee at Fishawack Scientific Communications Ltd., funded by GlaxoSmithKline.

Conflict of interest Denise A. Yardley, Lowell Hart, Linda Bosserman, Mansoor N. Salleh, David M. Waterhouse, Maura K. Hagan, and Paul Richards have no conflict of interest to declare. Michelle L. DeSilvio and Janine M. Mahoney are employees of, and own stock in, GlaxoSmithKline. Yasir Nagarwala is a former employee of GlaxoSmithKline and an employee of Celgene; he owns stock in GlaxoSmithKline and Celgene.

Open Access This article is distributed under the terms of the Creative Commons Attribution Noncommercial License which permits any noncommercial use, distribution, and reproduction in any medium, provided the original author(s) and the source are credited.

\section{References}

1. Siegel R, Naishadham D, Jemal A (2012) Cancer statistics, 2012. CA Cancer J Clin 62:10-29

2. Yarden Y, Sliwkowski MX (2001) Untangling the ErbB signalling network. Nat Rev Mol Cell Biol 2:127-137

3. Slamon DJ, Clark GM, Wong SG, Levin WJ, Ullrich A, McGuire WL (1987) Human breast cancer: correlation of relapse and survival with amplification of the HER-2/neu oncogene. Science 235:177-182

4. Slamon DJ, Godolphin W, Jones LA, Holt JA, Wong SG, Keith DE et al (1989) Studies of the HER-2/neu proto-oncogene in human breast and ovarian cancer. Science 244:707-712

5. Blows FM, Driver KE, Schmidt MK, Broeks A, van Leeuwen FE, Wesseling J et al (2010) Subtyping of breast cancer by immunohistochemistry to investigate a relationship between subtype and short and long term survival: a collaborative analysis of data for 10,159 cases from 12 studies. PLoS Med 7:e1000279

6. Mass RD, Press MF, Anderson S, Cobleigh MA, Vogel CL, Dybdal $\mathrm{N}$ et al (2005) Evaluation of clinical outcomes according to HER2 detection by fluorescence in situ hybridization in women with metastatic breast cancer treated with trastuzumab. Clin Breast Cancer 6:240-246

7. Gabos Z, Sinha R, Hanson J, Chauhan N, Hugh J, Mackey JR et al (2006) Prognostic significance of human epidermal growth 
factor receptor positivity for the development of brain metastasis after newly diagnosed breast cancer. J Clin Oncol 24:5658-5663

8. Kallioniemi OP, Holli K, Visakorpi T, Koivula T, Helin HH, Isola JJ (1991) Association of c-erbB-2 protein over-expression with high rate of cell proliferation, increased risk of visceral metastasis and poor long-term survival in breast cancer. Int $\mathbf{J}$ Cancer 49:650-655

9. Piccart-Gebhart MJ, Procter M, Leyland-Jones B, Goldhirsch A, Untch M, Smith I et al (2005) Trastuzumab after adjuvant chemotherapy in HER2-positive breast cancer. $N$ Engl J Med 353:1659-1672

10. Romond EH, Perez EA, Bryant J, Suman VJ, Geyer CE Jr, Davidson NE et al (2005) Trastuzumab plus adjuvant chemotherapy for operable HER2-positive breast cancer. N Engl J Med 353:1673-1684

11. Slamon DJ, Leyland-Jones B, Shak S, Fuchs H, Paton V, Bajamonde A et al (2001) Use of chemotherapy plus a monoclonal antibody against HER2 for metastatic breast cancer that overexpresses HER2. N Engl J Med 344:783-792

12. Montemurro F, Donadio M, Clavarezza M, Redana S, Jacomuzzi ME, Valabrega G et al (2006) Outcome of patients with HER2positive advanced breast cancer progressing during trastuzumabbased therapy. Oncologist 11:318-324

13. Geyer CE, Forster J, Lindquist D, Chan S, Romieu CG, Pienkowski $\mathrm{T}$ et al (2006) Lapatinib plus capecitabine for HER2positive advanced breast cancer. N Engl J Med 355:2733-2743

14. http://www.cancer.gov/cancertopics/druginfo/fda-lapatinib. Accessed 2 Oct 2012

15. Schwartzberg LS, Franco SX, Florance A, O'Rourke L, Maltzman J, Johnston S (2010) Lapatinib plus letrozole as first-line therapy for HER-2+ hormone receptor-positive metastatic breast cancer. Oncologist 15:122-129

16. Gradishar WJ, Tjulandin S, Davidson N, Shaw H, Desai N, Bhar $P$ et al (2005) Phase III trial of nanoparticle albumin-bound paclitaxel compared with polyethylated castor oil-based paclitaxel in women with breast cancer. J Clin Oncol 23:7794-7803

17. Gradishar WJ, Krasnojon D, Cheporov S, Makhson AN, Manikhas GM, Clawson A et al (2009) Significantly longer progression-free survival with $n a b$-paclitaxel compared with docetaxel as first-line therapy for metastatic breast cancer. J Clin Oncol 27:3611-3619

18. Gradishar WJ, Krasnojon D, Cheporov S, Makhson AN, Manikhas GM, Clawson A et al (2012) Phase II trial of nab-paclitaxel compared with docetaxel as first-line chemotherapy in patients with metastatic breast cancer: final analysis of overall survival. Clin Breast Cancer 12:313-321

19. http://www.cancer.gov/cancertopics/druginfo/fda-nanoparticle-paclitaxel. Accessed 2 Oct 2012

20. Gril B, Palmieri D, Bronder JL, Herring JM, Vega-Valle E, Feigenbaum L et al (2008) Effect of lapatinib on the outgrowth of metastatic breast cancer cells to the brain. J Natl Cancer Inst 100:1092-1103

21. Pestalozzi BC, Brignoli S (2000) Trastuzumab in CSF. J Clin Oncol 18:2349-2351

22. Xia W, Liu LH, Ho P, Spector NL (2004) Truncated ErbB2 receptor (p95ErbB2) is regulated by heregulin through heterodimer formation with ErbB3 yet remains sensitive to the dual EGFR/ErbB2 kinase inhibitor GW572016. Oncogene 23:646-653

23. Blackwell KL, Burstein HJ, Storniolo AM, Rugo HS, Sledge G, Aktan G et al (2012) Overall survival benefit with lapatinib in combination with trastuzumab for patients with human epidermal growth factor receptor 2-positive metastatic breast cancer: final results from the EGF104900 study. J Clin Oncol 30:2585-2592

24. Therasse P, Arbuck SG, Eisenhauer EA, Wanders J, Kaplan RS, Rubinstein L et al (2000) New guidelines to evaluate the response to treatment in solid tumors. European Organization for Research and Treatment of Cancer, National Cancer Institute of the United States, National Cancer Institute of Canada. J Natl Cancer Inst 92(205): 16

25. Greenwood M (1926) The natural duration of cancer. In: Reports on public health and medical subjects. London, England: His Majesty's Stationery Office, pp 1-26

26. Guan ZZ, Xu BH, DeSilvio M, Shen ZZ, Arponwirat W, Tong ZS et al (in press) Randomized trial of lapatinib vs placebo added to paclitaxel in the treatment of HER2-overexpressing metastatic breast cancer. J Clin Oncol

27. Jagiello-Gruszfeld A, Tjulandin S, Dobrovolskaya N, Manikhas A, Pienkowski T, DeSilvio M et al (2010) A single-arm phase II trial of first-line paclitaxel in combination with lapatinib in HER2-overexpressing metastatic breast cancer. Oncology 79: $129-135$

28. Di Leo A, Gomez HL, Aziz Z, Zvirbule Z, Bines J, Arbushites MC, et al (2008) Phase III, double-blind, randomized study comparing lapatinib plus paclitaxel with placebo plus paclitaxel as first-line treatment for metastatic breast cancer. J Clin Oncol 26:5544-5552. Erratum in: J Clin Oncol 2009;27:1923 